\title{
纳升电喷雾-离子迁移谱及其与高效液相色谱的联用
}

$$
\begin{aligned}
& \text { 陈 创 }{ }^{1,2} \text {, 王卫国 }{ }^{1} \text {, 梁莤莤 }{ }^{1,2} \text {, 周庆华 }{ }^{1,2} \text {, 彭丽英 }{ }^{1,2} \text {, } \\
& \text { 温 萌 }{ }^{1,2} \text {, 鞠帮玉 }{ }^{1} \text {, 赵 琨 }{ }^{1} \text {, 刘 军 }{ }^{3,4} \text {, 李海洋 }{ }^{1 *}
\end{aligned}
$$

(1. 中国科学院大连化学物理研究所, 辽宁 大连 $116023 ; 2$. 中国科学院大学, 北京 100049;

3. 南京财经大学, 江苏 南京 210000 ；４．江苏省质量安全工程研究院，江苏 南京 210000）

摘要: 搭建了一套纳升级电喷雾-离子源离子迁移谱仪。首先, 分别对尾吹气流速、溶剂流速等影响仪器去溶剂化效 果的参数进行了研究和优化。在此基础上, 用一系列胺类化合物对该仪器的去溶剂化效果、分辨能力以及灵敏度 进行了表征。实验结果表明, 该仪器能够对电喷雾离子液滴实现完全去溶剂化; 三辛胺的检出限可以达到 10 $\mu \mathrm{g} / \mathrm{L}$ 。最后, 将该仪器用作高效液相色谱的检测器, 在无需衍生化的条件下对胺类混合物样品进行检测。由三乙 胺、二乙胺以及丁胺组成的混合样品被成功分离并测定。该系统对三乙胺、二乙胺以及丁胺的线性响应范围均达 到近两个数量级。

关键词: 纳升电喷雾离子源; 离子迁移谱; 高效液相色谱;约化迁移率

中图分类号: O658 文献标识码:A 文章编号:1000-8713(2013)04-0386-06

\section{Nano-electrospray ionization-ion mobility spectrometry and its combination with high performance liquid chromatography}

\author{
CHEN Chuang ${ }^{1,2}$, WANG Weiguo ${ }^{1}$, LIANG Xixi ${ }^{1,2}$, ZHOU Qinghua ${ }^{1,2}$, PENG Liying ${ }^{1,2}$, \\ WEN Meng ${ }^{1,2}$, JU Bangyu ${ }^{1}$, ZHAO Kun ${ }^{1}$, LIU Jun ${ }^{3,4}$, LI Haiyang ${ }^{1 *}$ \\ (1. Dalian Institute of Chemical Physics, Chinese Academy of Sciences, Dalian 116023, China; \\ 2. University of Chinese Academy of Sciences, Beijing 100049, China; \\ 3. Nanjing University of Finance and Economics, Nanjing 210000, China; \\ 4. Jiangsu Province Institute of Quality \&Safety Engineering, Nanjing 210000, China)
}

\begin{abstract}
A nano-electrospray ionization-ion mobility spectrometer (nanoESI-IMS) was built up. Firstly, the effects of the parameters such as drift gas flow rate and solvent flow rate on the desolvation capability were studied and optimized. Then, a series of compounds were used to characterize the nanoESI-IMS system. The results showed that, complete desolvation was achieved for nano-electrospray ion droplets with the nanoESI-IMS apparatus. The limit of detection of this instrument for trioctylamine could reach $10 \mu \mathrm{g} / \mathrm{L}$. Finally, this instrument was coupled to the high performance liquid chromatography (HPLC) as a detector for amines analysis. A test mixture containing triethylamine, diethylamine and butylamine was successfully separated and determined by the HPLC-nanoESI-IMS system. Linear response ranges of about two orders of magnitude were achieved for triethylamine, diethylamine and butylamine with this system.
\end{abstract}

Key words : nano-electrospray ionization source; ion mobility spectrometry; high performance liquid chromatography (HPLC) ; reduced mobility

离子迁移谱( ion mobility spectrometry, IMS) 是 20 世纪 70 年代初出现的一种气相离子分离检测
技术 ${ }^{[1]}$ 。因具有灵敏度高、检测速度快、结构简单、 大气压条件下工作以及易于便携等优点, 已经被广

* 通讯联系人. Tel: (0411)84379510,E-mail:hli@ dicp. ac. cn.

基金项目: 国家高新技术研究发展计划 “ “863”计划)项目(2011AA060602); 国家自然科学基金项目 $(11004090$,20877074); 大连市 科技计划项目(2010J21DW028).

收稿日期: 2012-12-03 
泛应用在国土安全、环境污染、食品安全、生物医药 研究以及空间探索等领域 ${ }^{[2]}$ 。IMS 方法中, 待测样 品首先被离子化, 然后根据在弱电场 $(E / N<4 \mathrm{Td}$, $\left.1 \mathrm{Td}=10^{-17} \mathrm{~V} \cdot \mathrm{cm}^{2}\right)$ 中离子迁移率 $K$ 的不同对样 品不同组分进行分离和分析 ${ }^{[3]}$ 。其中折合电场强 度 $E / N$ 为电场强度与粒子数密度之比。在 IMS 仪 器中,使用最广泛的离子化源为放射性 ${ }^{63} \mathrm{Ni}$ 电离 源。这种离子源检测的样品主要是气体, 对于半挥 发性和难挥发的样品则需要特殊的热解吸进样器来 辅助进样, 极大地限制了 IMS 在热不稳定性样品和 生物样品分析中的应用。电喷雾离子源 ( electrospray ionization, ESI) 的出现, 使得 IMS 可以直接 对液体样品进行分析 ${ }^{[4]}$ 。随着仪器结构的不断改 进, 电喷雾电离-离子迁移谱 ( ESI-IMS) 被广泛应用 在环境污染物、违禁药品、医药、爆炸物以及生物样 品分析中。此外, ESI-IMS 也被作为多种液相分离 技术一一特别是高效液相色谱 ( high performance liquid chromatography, HPLC) 的检测器来使 用 $^{[5]}$ 。

传统 ESI-IMS 中, 电喷雾离子源中溶剂的流速 通常保持在 $1 \sim 100 \mu \mathrm{L} / \mathrm{min}$, 需要对电喷雾产生的 离子化小液滴进行复杂的去溶剂化处理, 以获得对 目标分析物好的分辨能力 ${ }^{[6,7]}$ 。通常要求 ESI-IMS 迁移管的温度升高到 $100{ }^{\circ} \mathrm{C}$ 以上, 并需要对电喷雾 离子源中的喷雾针进行特殊的冷却保护, 这在很大 程度上增加了仪器装置的复杂性, 不利于 ESI-IMS 与 HPLC 的联用; 另一方面, ESI-IMS 迁移管的温度 升高又会降低 ESI-IMS 的分辨能力 ${ }^{[8]}$ 。因此, 发展 能够在室温条件下实现很好去溶剂化效果的 ESIIMS 就显得非常重要。

1994 年, Wilm 和 Mann ${ }^{[9]}$ 发展了一种纳升级电
喷雾离子源 (nano-electrospray ionization source, nanoESI source)。与传统的电喷雾离子源相比, 这 种技术采用更小的溶剂流速 (10 500 nL/min), 形 成的喷雾更加稳定、喷雾产生的带电小液滴尺寸更 小 $(100 \sim 200 \mathrm{~nm})^{[10,11]}$ 。早期关于其机理的研究证 实了 nanoESI 中产生的小尺寸的电喷雾液滴在室 温的条件下就可以实现完全的去溶剂化 ${ }^{[12]}$, 这为我 们提供了一种降低 IMS 温度的有效方法。

本实验将 nanoESI 离子源用于 IMS 中, 搭建了 一套 nanoESI-IMS 装置。分别对尾吹气流速、溶剂 流速等影响 nanoESI-IMS 去溶剂化效果的参数进 行了研究和优化。在此基础上, 用一系列胺类化合 物对该仪器去溶剂化效果、分辨能力以及灵敏度进 行了表征。最后, 该 nanoESI-IMS 被用作 HPLC 的 检测器, 在无需衍生化的条件下对胺类混合物样品 进行分离和检测。

\section{1 实验部分}

\section{1 仪器、装置和试剂}

实验室搭建的 nanoESI-IMS 仪器装置如图 1 所示。IMS 主要包括纳升电喷雾电离源区、去溶剂 化腔体、离子门、离子迁移区以及法拉第盘检测器。 其中去溶剂化腔体的内径为 $14 \mathrm{~mm}$, 离子迁移区的 内径为 $25 \mathrm{~mm}$, 其他详细参数如表 1 所示。纳升电 喷雾离子源的喷雾针为毛细管 $(360 \mu \mathrm{m}$ o. d. , 200 $\mu \mathrm{m}$ i. d. ) 拉制而成, 喷雾针针尖外径 $10 \mu \mathrm{m}$, 内径 8 $\mu \mathrm{m}$ 。在表征 nanoESI-IMS 性能的时候, 配备有 3 $\mathrm{mL}$ 注射器的步进进样器 ( LSP01-1A/2A Longer Pump), 用于将电喷雾溶剂输送到纳升喷雾的喷雾 针中进行电喷雾。溶剂的流速设定为 $200 \mathrm{~nL} / \mathrm{min}$, 并且可以通过步进进样器进行调节。电喷雾高压通

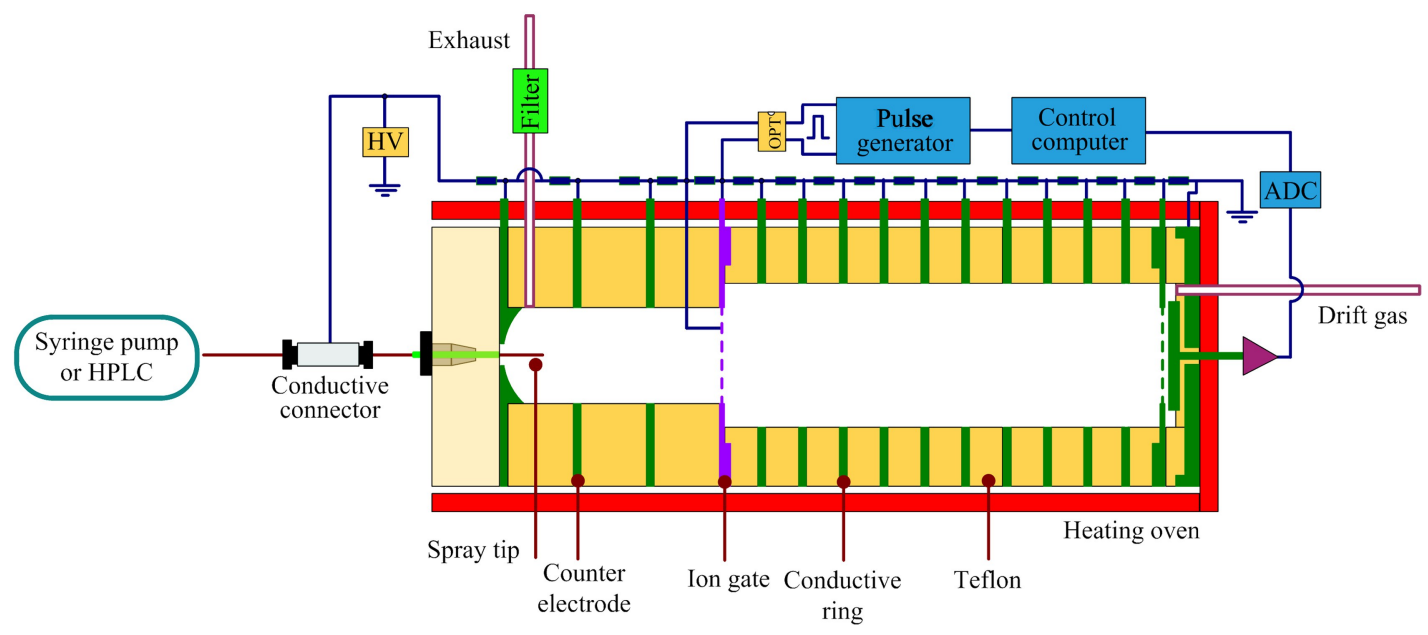

图 1 纳升电喷雾-离子迁移谱仪的结构示意图 
过导电两通传导到电喷雾溶剂中。样品溶液经过电 喷雾后,形成的离子在去溶剂化腔体中进行去溶剂 化, 然后在离子门脉冲作用下进人 IMS 迁移区进行 分离。分离后的离子最终到达法拉第盘被检测。 nanoESI-IMS 的迁移管的温度保持在 $32{ }^{\circ} \mathrm{C}$ 。

表 1 IMS 相关参数

Table 1 The parameters of IMS

\begin{tabular}{ll}
\hline \multicolumn{1}{c}{ Parameter } & \multicolumn{1}{c}{ Value } \\
\hline IMS mode & positive ion mode \\
Desolvation chamber length & $48 \mathrm{~mm}$ \\
Drift region length & $88 \mathrm{~mm}$ \\
Electric field strength & $250.25 \mathrm{~V} / \mathrm{cm}$ \\
Shutter pulse width & $200 \mu \mathrm{s}$ \\
Drift gas & purified air \\
Nano-electrospray solvent flow rate & $180 \mathrm{~nL} / \mathrm{min}$ \\
Nano-electrospray voltage & $4000 \mathrm{~V}$ \\
\hline
\end{tabular}

实验中使用的高效液相色谱仪为 Jasco HPLC $\operatorname{system}($ PU-1580)。色谱柱为 Waters C18 色谱柱 $\left(100 \mathrm{~mm} \times 4.6 \mathrm{~mm}, 5 \mu \mathrm{m}, \mathrm{XTerra}^{\mathrm{TM}}\right)$ 。当配接 HPLC 到 nanoESI-IMS 时,色谱柱的流出物经过一 个三通连接头 $($ P-888, Upchurch Scientific, 其中两 端流阻不同)进行分流, 大约以 $200 \mathrm{~nL} / \mathrm{min}$ 的流速 被输送到 nanoESI-IMS 进行分析。流速的大小通 过控制三通连接头两端流阻的大小来实现, 流量的 估测使用带有刻度的玻璃毛细管 ( P-015, Upchurch Scientific)进行。

所用试剂甲醇、二乙胺、三乙胺、丁胺、三辛胺和 2,4-二甲基吡啶均为高效液相色谱纯级别, 购买自 天津 Kermel 公司。所使用的水为去离子化超纯水 (18 $\mathrm{M} \Omega \cdot \mathrm{cm}$, Milli-Q, Advantage-A10)。

\section{2 标准溶液的配制}

实验过程中使用 $80 \%$ （如无特殊说明均为体积 分数) 甲醇作为溶剂配制所需的二乙胺、三乙胺、丁 胺、三辛胺和 2,4-二甲基吡啶样品母液, 质量浓度 均为 $1 \mathrm{~g} / \mathrm{L}_{\circ}$ 实验过程中所需要的不同浓度样品溶 液均通过逐级稀释方法获得。在每次实验前,均重 新配制新鲜样品母液。

\section{3 色谱分离条件}

高效液相色谱使用等度洗脱模式, 流动相为 $90 \%$ 甲醇溶液, 流动相流速为 $0.8 \mathrm{~mL} / \mathrm{min}$ 。每次进 样量为 $5 \mu \mathrm{L}$ 。

\section{4 约化迁移率和分辨能力}

所有化合物的约化离子迁移率 $K_{\mathrm{o}}$ 均通过它们 在 nanoESI-IMS 中形成的产物离子峰的迁移时间 $t_{\mathrm{d}}$ 来计算得到。公式 ${ }^{[3]}$ 为

$$
K_{\mathrm{o}}=\frac{L}{E \times t_{\mathrm{d}}} \times \frac{273}{T} \times \frac{P}{760}
$$

其中, $L$ 表示离子迁移区的长度 $(8.8 \mathrm{~cm}), E$ 表示 离子迁移区电场强度 $(250.25 \mathrm{~V} / \mathrm{cm}), T$ 表示迁移 区的温度 $(305 \mathrm{~K}), P$ 为 nanoESI-IMS 内部的压强 (101325 Pa)。

所有化合物的分辨能力 $R_{\mathrm{p}}$ 均通过下面公式 ${ }^{[3]}$ 计算:

$$
R_{\mathrm{p}}=\frac{t_{\mathrm{d}}}{w_{1 / 2}}
$$

其中, $w_{1 / 2}$ 为产物离子峰峰高一半处的峰宽。

\section{2 结果与讨论}

\section{1 纳升电喷雾-离子迁移谱性能的考察}

去溶剂化的效果很大程度上会影响 nanoESIIMS 性能, 包括其分辨能力以及灵敏度。因此, 首先 对影响去溶剂化效果的因素包括尾吹气的流速、电 喷雾溶剂的流速等条件进行了考察和优化。在此基 础上, 通过检测不同化合物来对 nanoESI-IMS 去溶 剂化的效果进行评价, 同时对 nanoESI-IMS 分辨能 力和灵敏度进行考察。

\section{1 .1 尾吹气流速的影响}

以 $80 \%$ 的甲醇作为电喷雾溶剂, 记录了不同尾 吹气流速下 nanoESI-IMS 的响应谱图, 并将其中主 要溶剂离子峰的位置随尾吹气流速变化的趋势作 图, 结果如图 2。尾吹气流速为 $10 \mathrm{~mL} / \mathrm{min}$ 的时候, 甲醇溶液在 nanoESI-IMS 中形成的溶剂离子峰具 有非常长的迁移时间 (17.046 ms)。随着尾吹气流 速的增大, 溶剂离子峰的迁移时间迅速变短, 这种趋 势一直持续到尾吹气流速增加到 $350 \mathrm{~mL} / \mathrm{min}_{\text {。 }}$ 这 一迁移时间减小的变化是由于随着尾吹气流速的增 大,nanoESI 产生的离子液滴中的溶剂蒸发速度加 快,生成质量更小、迁移速度更快的离子 ${ }^{[7]}$ 。而随 着尾吹气流速进一步增大, 溶剂离子峰开始缓慢向 迁移时间长的方向移动, 从 $350 \mathrm{~mL} / \mathrm{min}$ 时的 13.53

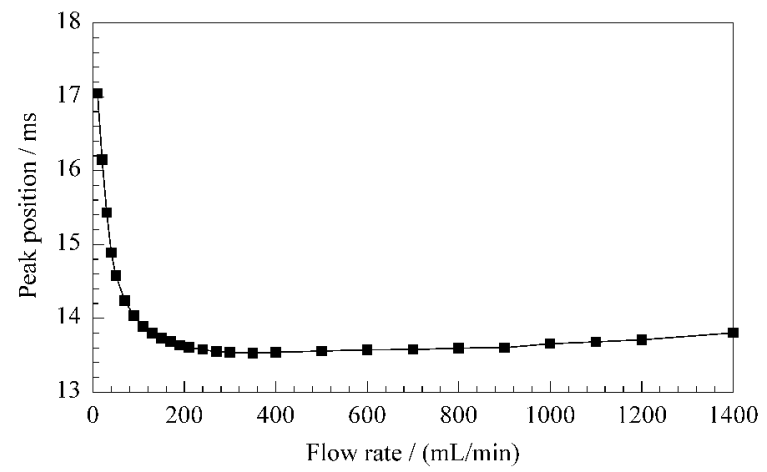

图 2 尾吹气流速对主溶剂峰位置的影响

Fig. 2 Effect of drift gas flow rate on the position of major solvent ion peak 
$\mathrm{ms}$ 至 $900 \mathrm{~mL} / \mathrm{min}$ 时的 $13.60 \mathrm{~ms}$ 。并且随着尾吹 气流速进一步增加, 其迁移时间开始向着更长的方 向迅速移动, 从 $900 \mathrm{~mL} / \mathrm{min}$ 时的 $13.60 \mathrm{~ms}$ 至 $1400 \mathrm{~mL} / \mathrm{min}$ 时的 $13.80 \mathrm{~ms}$ 。这一增大的趋势主 要是由于随着气流流速增大, 迁移管内部压力增大, 对溶剂离子的运动阻力增大, 导致溶剂离子运动速 度变慢 ${ }^{[13]}$ 。大的尾吹气流速对保持离子迁移谱内 部清洁非常重要, 同时考虑到其对溶剂蒸发的影响, 在后续实验中将尾吹气流速设定在 $900 \mathrm{~mL} / \mathrm{min}_{\text {。 }}$

\subsection{2 溶剂流速的影响}

纳升电喷雾产生的带电离子液滴的半径 $R$ 与 溶剂的流速 $v$ 之间存在着这样的关系 $R \propto v^{2 / 3[14] 。}$ 因此, 在电喷雾电压固定的条件下, 溶剂的流速越 大, 纳升电喷雾产生的带电离子液滴的半径就越大。 半径越大, 其中溶剂完全蒸发就越困难。为了研究 不同溶剂流速对 nanoESI-IMS 去溶剂化能力的影 响,采集了 $80 \%$ 甲醇在不同流速下的离子迁移谱 图, 并将其溶剂离子峰的位置随着流速变化趋势作 图, 结果如图 3 。溶剂流速从 $60 \mathrm{~nL} / \mathrm{min}$ 变化到 $1560 \mathrm{~nL} / \mathrm{min}$ 的过程中, 溶剂离子峰的位置始终保 持在 $13.60 \mathrm{~ms}$ 不变。这一结果反映了该 nanoESIIMS 在此溶剂流速区间内能够保持恒定去溶剂化效 果, 同时也为后续实验中将 nanoESI-IMS 配接到 HPLC 上提供了一个较宽的流速变化范围。为了获 得更好的去溶剂化效果和方便将 nanoESI-IMS 配 接到 HPLC 上, 选择溶剂流速 $200 \mathrm{~nL} / \mathrm{min}$ 左右。

\subsection{3 纳升电喷雾-离子迁移谱的去溶剂化效果}

在上述优化条件下,使用 nanoESI-IMS 获得的 2,4-二甲基吡啶谱图如图 4。图 4 中, 除了甲醇溶液 产生的溶剂离子峰以外, 可以观察到明显的 2,4-二 甲基吡啶的产物离子峰, 峰位置在 $15.80 \mathrm{~ms}$ 处。利 用公式(1) 计算得到约化迁移率 $K_{\mathrm{o}}$ 为 $1.99 \mathrm{~cm}^{2} /$ $(\mathrm{V} \cdot \mathrm{s})$ (见表 2)。根据文献 ${ }^{[7]}$ 报道, 如果 ESI-IMS 检测 2,4-二甲基吡啶得到的约化迁移率值小于 $1.95 \mathrm{~cm}^{2} /(\mathrm{V} \cdot \mathrm{s})$, 则表示 2,4 -二甲基吡啶离子的 去溶剂化不完全。而本实验中得到的约化迁移率值 与文献 ${ }^{[7]}$ 中报道的 $1.95 \mathrm{~cm}^{2} /(\mathrm{V} \cdot \mathrm{s})$ 相吻合, 表示 在该 nanoESI-IMS 装置中 2,4-二甲基吡啶离子得

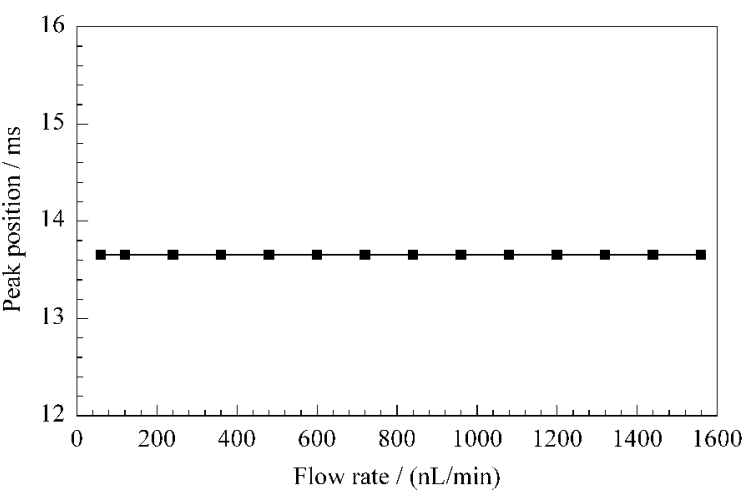

图 3 电喷雾溶剂流速对主溶剂峰位置的影响

Fig. 3 Effect of nano-electrospray solvent flow rate on the position of major solvent ion peak

到了完全去溶剂化。表 2 中一同列出了二乙胺、三 乙胺、丁胺和三辛胺的约化迁移率, 并给出了文献中 报道的迁移率。这些相互吻合的迁移率值反映了该 nanoESI-IMS 仪器在室温下同样具有较好的去溶剂 化能力。利用公式(2)得到的各化合物的分辨能力 值也列在表 2 中, 分辨能力值在 33 到 40 之间, 与文 献中利用传统的 ESI-IMS 在高的迁移管温度下 $\left(100{ }^{\circ} \mathrm{C}\right)$ 得到的结果一致 ${ }^{[7]}$ 。

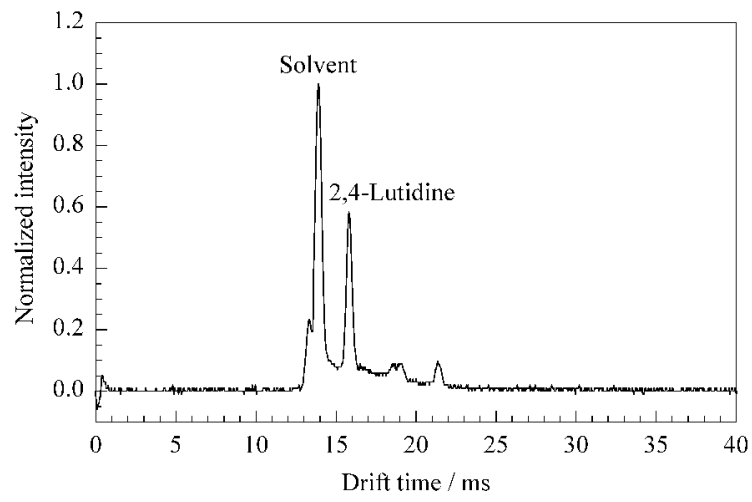

图 42 , 4-二甲基吡啶的 nanoESI-IMS 离子迁移谱图 Fig. 4 NanoESI-IMS spectrum for 2 ,4-lutidine

\subsection{4 纳升电喷雾-离子迁移谱的灵敏度}

图 5 展示了 $60 \mu \mathrm{g} / \mathrm{L}$ 三辛胺样品在 nanoESIIMS 中的响应谱图。在谱图中迁移时间为 30.08 $\mathrm{ms}$ 处观察到三辛胺的离子峰。该离子峰的强度为 $11.8 \mathrm{mV}$, 基线噪声水平为 $0.38 \mathrm{mV}$, 计算得到 $S / N$ $=31$ 。为了考察 nanoESI-IMS 检测三辛胺的检出

表 2 五种化合物在 nanoESI-IMS 中的分辨能力以及约化迁移率

Table 2 Resolving power and reduced mobility $\left(K_{\mathrm{o}}\right)$ for the five compounds in nanoESI-IMS

\begin{tabular}{|c|c|c|c|c|c|}
\hline Compound & $M_{\mathrm{w}} / \mathrm{Da}$ & Drift time/ms & Resolving power & $K_{\mathrm{o}} /\left(\mathrm{cm}^{2} /(\mathrm{V} \cdot \mathrm{s})\right)$ & Literature $K_{\mathrm{o}} /\left(\mathrm{cm}^{2} /(\mathrm{V} \cdot \mathrm{s})\right)$ \\
\hline 2,4 -Lutidine & 107 & $15.80 \pm 0.02$ & 36.71 & 1.99 & $1.95^{[4]}$ \\
\hline Diethylamine & 73 & $14.64 \pm 0.02$ & 33.51 & 2.15 & $2.16^{[15]}$ \\
\hline Butylamine & 73 & $15.82 \pm 0.02$ & 36.88 & 1.99 & $1.98^{[15]}$ \\
\hline Triethylamine & 101 & $15.38 \pm 0.02$ & 37.42 & 2.05 & $1.96^{[15]}$ \\
\hline Trioctylamine & 355 & $29.92 \pm 0.02$ & 40.51 & 1.05 & $0.92^{[6]}$ \\
\hline
\end{tabular}


限,利用逐级稀释方法依次记录了 40 、20 和 10 $\mu \mathrm{g} / \mathrm{L}$ 三辛胺样品在 nanoESI-IMS 中的响应信号强 度。发现三辛胺质量浓度为 $10 \mu \mathrm{g} / \mathrm{L}$ 时响应信号强 度与噪声水平的比值为 3.2 , 接近 $S / N=3$ 的水平。 因此我们将 $10 \mu \mathrm{g} / \mathrm{L}$ 作为 nanoESI-IMS 检测三辛 胺的检出限。这一数值达到 $\mathrm{Jafari}^{\left[{ }^{[6]}\right.}$ 利用传统的 ESI-IMS 得到的检出限范围 ( $5 \sim 40 \mu \mathrm{g} / \mathrm{L}$ ), 表明了 该 nanoESI-IMS 同样具有高的灵敏度。

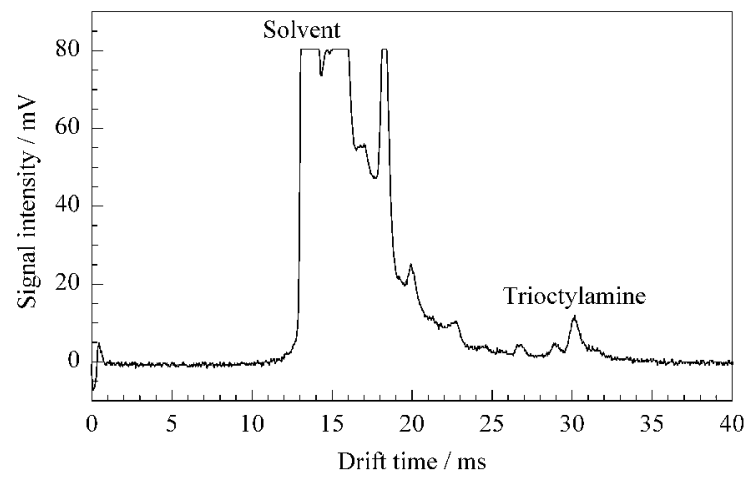

图 $560 \mu \mathrm{g} / \mathrm{L}$ 的三辛胺的 nanoESI-IMS 谱图

Fig. 5 NanoESI-IMS spectrum for trioctylamine of $60 \mu \mathrm{g} / \mathrm{L}$

\section{2 高效液相色谱与纳升电喷雾-离子迁移谱联用}

\subsection{1 二维分离检测}

在考察了 nanoESI-IMS 性能的基础上, 将 nanoESI-IMS 配接到高效液相色谱仪上, 对低质量 数脂肪胺化合物进行无衍生化处理的分离和检测。 图 6 是使用 HPLC-nanoESI-IMS 系统检测二乙胺、 三乙胺和丁胺混合物得到的二维等高线图,其中的 色谱图为总离子色谱图 (将迁移时间在 $14 \mathrm{~ms}$ 到 20 $\mathrm{ms}$ 之间的各个质谱图的离子强度加和得到)。从 图 6 可以看出,这 3 种胺的色谱分离效率并不高, 仅 仅观察到两个色谱峰。这是由于二乙胺和丁胺在色 谱柱中的保留时间基本相同,两者形成的离子峰重 合在一起成为一个峰。但 nanoESI-IMS 在作为 HPLC 检测器的同时, 又提供了迁移时间维度的分 离。从图 6 二维等高线图中可以观察到 3 个相互完 全分离的离子峰(标有 $1 、 2$ 和 3 的 3 个点)。这一结 果展示了这种二维分离手段在分离能力互补方面的 特性。

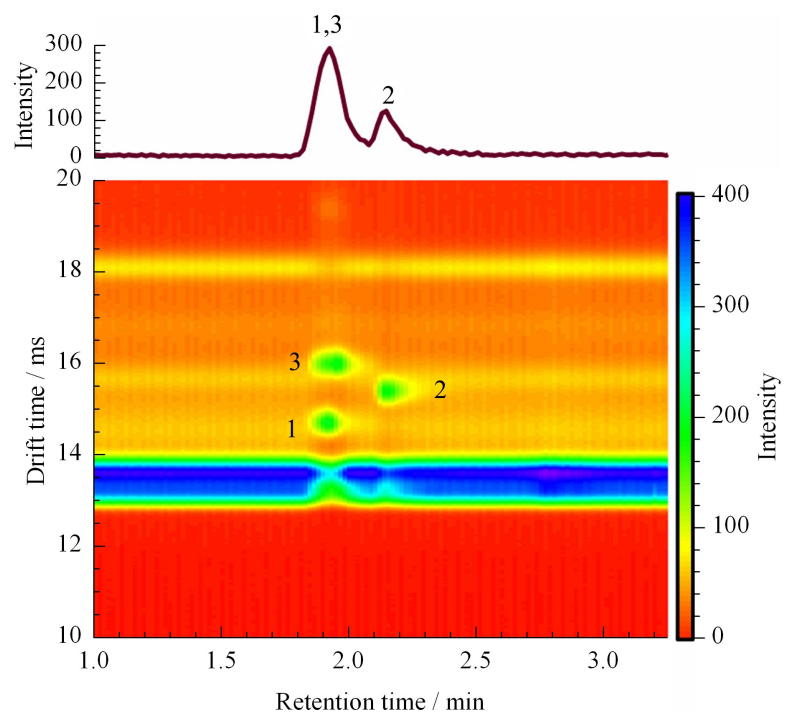

图 6 HPLC-nanoESI-IMS 系统分离分析二乙胺、 三乙胺和丁胺混合物得到的二维等高线图和 对迁移时间 $14 \mathrm{~ms}$ 到 $20 \mathrm{~ms}$ 之间的离子强度 求和得到的总离子色谱图

Fig. 6 Two-dimensional contour plot for a mixture of diethylamine, triethylamine and butylamine and total ion chromatogram of summed ion intensities from $14 \mathrm{~ms}$ to $20 \mathrm{~ms}$ obtained with the HPLC-nanoESI-IMS system

Peaks: 1. diethylamine; 2 . triethylamine; 3 . butylamine.

\subsection{2 定量分析}

验证了 HPLC-nanoESI-IMS 系统的分离检测能 力后, 对该系统的定量分析能力进行评价。测试了 该系统对二乙胺、三乙胺和丁胺的线性响应范围。 其中,样品产物离子中心迁移时间对应的色谱图的 峰面积被用来作为样品信号响应值, 用于绘制样品 的浓度响应曲线,结果见表 3 。可以看到该系统对 于这 3 种脂肪胺的线性响应范围都达到 2 个数量 级, 并且相关系数都达到 0.995 以上。此外, 以 $S / N$ $=10$ 确定上述 3 种胺的定量限分别为 $0.8 、 1$ 和 0.8 $\mathrm{mg} / \mathrm{L}_{\text {。 }}$ 其值明显高于文献 ${ }^{[16]}$ 中使用化学发光法进 行高效液相色谱检测的结果 $(7 \sim 13 \mu \mathrm{g} / \mathrm{L})$ 。这可 能是由于使用了分流模式, 分析物流出液相色谱柱 后只有一小部分进人到 nanoESI-IMS 中进行分析。 在下一步的实验中,将采用毛细管色谱柱来进行高 效液相色谱分离, 尝试提高检出限。

表 3 HPLC-nanoESI-IMS 系统检测二乙胺、三乙胺和丁胺的分析参数

Table 3 Analytical parameters for triethylamine, diethylamine and butylamine using HPLC-nanoESI-IMS

\begin{tabular}{cccccc}
\hline Compound & Linear function & Linear range/ $(\mathrm{mg} / \mathrm{L})$ & $R^{2}$ & $\mathrm{LOQ} /(\mathrm{mg} / \mathrm{L})$ & $\mathrm{RSD} / \%(n=3)$ \\
\hline Diethylamine & $y=0.43+0.94 x$ & $0.5-100$ & 0.996 & 0.8 & 4 \\
Triethylamine & $y=0.48+0.88 x$ & $1-100$ & 0.995 & 1 & 3 \\
Butylamine & $y=0.64+0.82 x$ & $0.8-100$ & 0.998 & 0.8 & 4 \\
\hline
\end{tabular}

$y$ : peak area; $x$ : sample concentration, $\mathrm{mg} / \mathrm{L} ; R^{2}$ : correlation coefficient. 


\section{3 结论}

成功搭建了一套 nanoESI-IMS 系统, 对其性能 进行了考察, 并将其用作 HPLC 系统的检测器对一 些低质量数的胺类化合物进行了初步的检测和定量 分析。由于传统的高效液相色谱使用高的流动相流 速, 在两者配接的过程中只有很小一部分的色谱柱 流出物进样到 nanoESI-IMS 中进行分析, 对分析样 品的利用率极低。另一方面,这种配接方式会造成 色谱峰在传输过程中的展宽, 从而造成分离效率的 下降和灵敏度的降低。当前毛细管液相色谱的发 展 ${ }^{[17]}$, 为这种两维分离手段结合提供了一种新的技 术改进方案。因为毛细管色谱柱使用的流动相的流 速低, 可以低到 $200 \mathrm{~nL} / \mathrm{min}$ 左右, 非常适合于和 nanoESI-IMS 的配接,不需要分流的处理,而且色谱 柱和 nanoESI 喷雾针的连接将更加的方便。在后 续的研究中,将毛细管整体柱和 nanoESI-IMS 联用 将成为研究的重点。

\section{参考文献:}

[1] Xu F, Wang H L, Guan Y F. Progress in Chemistry (许峰, 王海龙, 关亚风. 化学进展), 2005, 17: 514
[2] Borsdorf H, Mayer T, Eiceman G A. Appl Spectrosc Rev, 2011, 46: 472

[3] Eiceman G A, Karpas Z. Ion Mobility Spectrometry. 2nd ed. Boca Raton, Florida: CRC Press, 2005

[4] Kanu A B, Kumar B S, Hill H H. Int J Ion Mobil Spectrom, 2012, 15: 9

[5] Kurnin I V, Kayumov A A, Muradymov M Z. Int J Ion Mobil Spectrom, 2012, 15: 51

[6] Jafari M. Talanta, 2009, 77: 1632

[7] Jafari M T, Javaheri M. Anal Chem, 2010, 82:6721

[8] Matz L M, Steiner W E, Hill H H. Int J Mass Spectrom, 2002, 213: 191

[9] Wilm M, Mann M. Anal Chem, 1996, 68:1

[10] Kebarle P, Verkerk U H. Mass Spectrom Rev, 2009, 28 : 898

[11] Heemskerk A, Hensbergen P J, Mayboroda O A. Anal Chem, 2012, 84 : 4552

[12] Crotti S, Seraglia R, Traldi P. Eur J Mass Spectrom, 2011, $17: 85$

[13] Kwasnik M, Fuhrer K, Fernandez F M. Anal Chem, 2007, 79: 7782

[14] Wilm M S, Mann M. Int J Mass Spectrom Ion Processes, 1994, 136: 167

[15] Karpas Z. Anal Chem, 1989, 61: 684

[16] Lloret S M, Legua C M, Falco P C. J Chromatogr A, 2004, $1035: 75$

[17] Gaspari M, Cuda G. Methods Mol Biol, 2011, 790: 115 